\title{
Differences in cue-dependent spatial navigation may be revealed by in-depth swimming analysis
}

\author{
Deirdre R. Harvey, Lesley Brant, Sean Commins* \\ Department of Psychology, National University of Ireland Maynooth, Maynooth, Co. Kildare, Ireland
}

\section{A R T I C L E I N F O}

\section{Article history:}

Received 9 February 2009

Received in revised form 10 June 2009

Accepted 22 June 2009

\section{Keywords:}

Allocentric

Spatial navigation

Cues

Water maze

\begin{abstract}
A B S T R A C T
Several factors can influence allocentric navigation in the Morris water maze (MWM), including the number of available distal visual cues. Using in-depth analytical measures investigating platform-based and swimming behaviour, we examine and compare animals exposed to either one or three distal visual cues during MWM acquisition. We demonstrate that, although animals exposed to one cue can acquire the task as well as those in a multiple cue condition, several subtle differences between the groups' swimming behaviours are noted. Both groups actively use cues to guide them to the platform, but changing the number of cues alters the animals' patterns of behaviour, wherein exposure to a single cue leads to a simpler strategy in which the cue appears to act as a beacon for navigation.
\end{abstract}

(C) 2009 Elsevier B.V. All rights reserved.

\section{Introduction}

Animals have a propensity to rely on external distal visual cues during navigation in the Morris water maze (MWM, Morris, 1981, 1984; McGauran et al., 2004; Harvey et al., 2008; Kealy et al., 2008). Previous research indicates that there are several influences on spatial navigation, including the number of visual cues available, the geometric arrangement of cues and the shape of the testing environment (Fenton et al., 1994; Prados, 2000; Prados and Trobalon, 1998; Hayward et al., 2004). To locate a goal with reference to a single landmark, an animal has to recognise the distance and direction of their own position with respect to this landmark, to remember the distance and direction between the landmark and the goal, and to then implicitly calculate the distance and direction from their current position to the goal. While a single beacon is sufficient to indicate the location of a goal, a single symmetrical landmark is not. Such a landmark only indicates the distance to the goal, but not the direction. Nevertheless, it is still possible to find a goal using only one distal landmark. Cartwright and Collett (1983) have shown that honeybees can learn to find a sucrose solution in a cup located centimetres from a black cylinder cue (both distance and direction were fixed). Once the bees learned to fly directly to the sucrose they were tested by removing the sucrose. Researchers observed the bees persistently search in the correct location. In another test, when the cylinder was removed it was shown that bees failed to search near the goal, indicating that it was employed as a landmark for the sucrose. Collett et al. (1986) carried out a comparable exper-

\footnotetext{
* Corresponding author. Tel.: +353 1 7084765; fax: +3531 1084767 .

E-mail address: Sean.Commins@nuim.ie (S. Commins).
}

iment with gerbils where they learned to dig for a sunflower seed buried under black granite chips, with only a single cylindrical landmark located a fixed distance $(50 \mathrm{~cm})$ and direction away. As with honeybees, the gerbils learned to search in the correct region based on the sole landmark.

More specifically, when it comes to the MWM task, animals have been shown to acquire the task in certain circumstances with one distal visual cue available (Chamizo and Rodrigo, 2004; Roberts and Pearce, 1999). Chamizo and Rodrigo (2004), for example, showed that the distance the landmark was from the goal was a key factor in the animals' ability to acquire the task. Trained rats were more impaired when the available cue was suspended on the wall on the opposite side of the pool than when it was positioned on the wall near the hidden platform. However, the animals' performance was best when the cue was suspended directly above the platform itself, therefore being used in a beaconing fashion, by simply moving towards it (Gallistel, 1990). Similar suggestions have been made whereby the absolute distance between the single landmark and the hidden platform have a significant effect on how well it is employed in locating the platform, with closer positioning resulting in better performance (Chamizo and Rodrigo, 2004; Chamizo et al., 2006; Spetch, 1995). A sole distal cue however cannot unambiguously define the location of the platform; it can only at most give information on the distance of the platform from the pool wall. Animals do not have directional information at their disposal.

When multiple cues are located in a consistent relationship to each other and to the goal animals can very effectively navigate towards this goal. In an early study conducted by Collett et al. (1986) it was clearly demonstrated that animals did not treat multiple landmarks independently but rather in a configural fashion. Indeed, if rats are trained in the MWM to find a hidden platform 
that is indicated by four landmarks, they tend to learn about all four, even though they are capable of finding the goal with just two (Prados and Trobalon, 1998; Rodrigo et al., 1997). For example, in the Rodrigo et al. (1997) study, following water maze training with four landmarks animals were tested without the platform in the presence of two or three landmarks. Results indicated that there was no difference in performance in the presence of two or three landmarks and animals searched in the area of where the platform had been during training (Experiment 1A). In Experiment $1 \mathrm{~B}$ of this study the authors then tested animals in the presence of two landmarks and with one landmark presented one at a time. Animals, in the presence of two landmarks, preferentially searched in the platform area but animals, in the presence of one landmark did not. These results suggest that no specific landmark is necessary for successful performance on the watermaze and that animals solve the task by learning a configuration of landmarks rather than each landmark individually. Similar results have been found by Fenton et al. (1994). In their study, rats were trained with either two or four cues, with those in the 2-cues group performing as well as those in the 4-cues group. It was also shown that animals could use any two subsets of the four cues to support spatial learning. However animals, in this study, were unable to use only one familiar cue for successful spatial navigation in test trials. Thus, in a multiple cue situation, it is generally accepted that animals identify the position of the platform by referring to the geometric arrangement of landmarks to the hidden platform (Benhamou and Poucet, 1998; Collett et al., 1986; Gallistel and Cheng, 1985; Maurer and Derivaz, 2000; Roberts and Pearce, 1999) rather than one lone landmark.

It has been recently demonstrated that several swimming behaviours are used by animals when locomoting in the MWM, all of which seem to incorporate the available distal cues (Harvey et al., 2008). We found, using in-depth analysis of swimming activity, that animals head directly towards the cues, move their bodies in a scanning motion whilst swimming towards a cue and turn at certain locations around the pool that seem to be influenced by the available cues. As an animal learns the task, some or all of these swimming behaviours change. This analytical approach may potentially be useful in the examination of how animals respond to different environments and may provide an insight into the different strategies that animals may employ during learning. As an initial examination, we aim to determine the effect of reduced cue availability on the pattern of swimming behaviour. If animals actively use the cues during navigation (as suggested by Harvey et al., 2008), then, by altering the number of cues that are available to them during acquisition differences in some of the swimming patterns should be observed. In this experiment we analyse the swimming pattern of animals as they search for the hidden platform in an environment containing three cues compared to an environment containing a single cue. We suggest that the single cue will act like a beacon and predict that animals will approach this cue significantly more than a cue in a similar position but part of a multi-cued configuration. Likewise animals will scan towards the single cue significantly more than towards an individual cue of the 3-cues array, further we predict that their range of scanning will be reduced. We finally predict that the location of turns towards the cue in the 1-cue group will be confined to a more precise location compared to the 3 -cues group.

\section{Methods}

\subsection{Animals}

Male Wistar naïve rats (250-350 g; Biomedical Facility, University College Dublin) aged approximately 3 months were used as subjects. Rats were housed three per cage and were kept in a temperature-controlled room that was maintained on a fixed 12:12 h light-dark cycle. All rats were given free access to food and drink. All rats were well handled before experimentation and all testing was carried out during the light phase. Guideline for the maintenance and experimentation of animals conformed to the Department of Health and Children (Ireland) guidelines and European directive 86/609/EC.

\subsection{Apparatus}

The Morris water maze, a black circular fibreglass pool $(1.7 \mathrm{~m}$ diameter; $38 \mathrm{~cm}$ deep), was filled with water to a depth of approximately $31 \mathrm{~cm}$ and kept at a temperature of $20 \pm 1^{\circ} \mathrm{C}$. Rats could escape the water by locating a hidden concrete platform $(9 \mathrm{~cm}$ diameter, $29 \mathrm{~cm}$ height) located in all experiments in the northeast quadrant of the pool. The platform was submerged $2 \mathrm{~cm}$ below the water surface, rendering it invisible to the rats when swimming. The pool was surrounded by a black curtain which was located approximately $50 \mathrm{~cm}$ from the pool wall. Distal cues used included a 40 and a $60 \mathrm{~W}$ light bulb, both were suspended from the ceiling. One bulb was located in the northwest corner (NW) and the other in the northeast (NE) corner. Both lights were located on the inside of the curtain at a distance of $75 \mathrm{~cm}$ from the pool at an angle of approximately $60^{\circ}$. A rectangular sheet of white paper $(55 \mathrm{~cm} \times 81 \mathrm{~cm})$ was also attached to the curtain on the east side of the pool for use as a cue. Different numbers of cues were available for different groups (see below and Fig. 1a, inset). All movements and escape latencies of each animal were recorded by using the EthoVision tracking system (Noldus Information Technologies, Wageningen, Netherlands).

\subsection{Procedure}

All animals ( $n=15)$ were given 4 trials/day for 5 days to locate the hidden platform. Training occurred in the light for all animals. All animals were placed into the water facing the pool wall from one of four pseudorandom starting positions (north, south, east or west). All animals were allowed $60 \mathrm{~s}$ to locate the platform. If an animal did not locate the platform within this period, it was led to the platform by the experimenter. All animals were then allowed $15 \mathrm{~s}$ to remain on the platform before commencing their next trial. Following each trial, animals were given approximately a $15 \mathrm{~s}$ intertrial interval where animals were kept in an open-topped box. Prior to acquisition animals were randomly assigned to one of two groups. For the first group (1-cue group, $n=8$ ), only one cue was available. This was the $60 \mathrm{~W}$ light cue positioned in the NE corner of the pool (see Fig. 1a, left inset). For the second group (3-cues group, $n=7$ ), three distal cues were used (light bulb NW corner, light bulb NE corner, and the white rectangular card, see Fig. 1a, right inset).

\subsection{Head direction analysis}

All animals, while sitting on the platform, were digitally recorded using an overhead camera with video capture software (VirtuaDub 1.5 10). This $15 \mathrm{~s}$ recorded interval was then segmented using TMPGEnc 2.5 (Hiroyuki Hori/Pegasys Inc.) to produce 15 digital photographs ( $1 \mathrm{~s}$ apart) for each trial for each animal $(n=5400$ total). From each photograph the head direction was calculated using Adobe Photoshop 5.0, based on a line running from the midpoint between the animal's eyes to the tip of the snout. Using the most southern point on the platform as $0^{\circ}$, the mean head direction of each animal on each trial was plotted and subsequently analysed using circular statistics. 
(a)
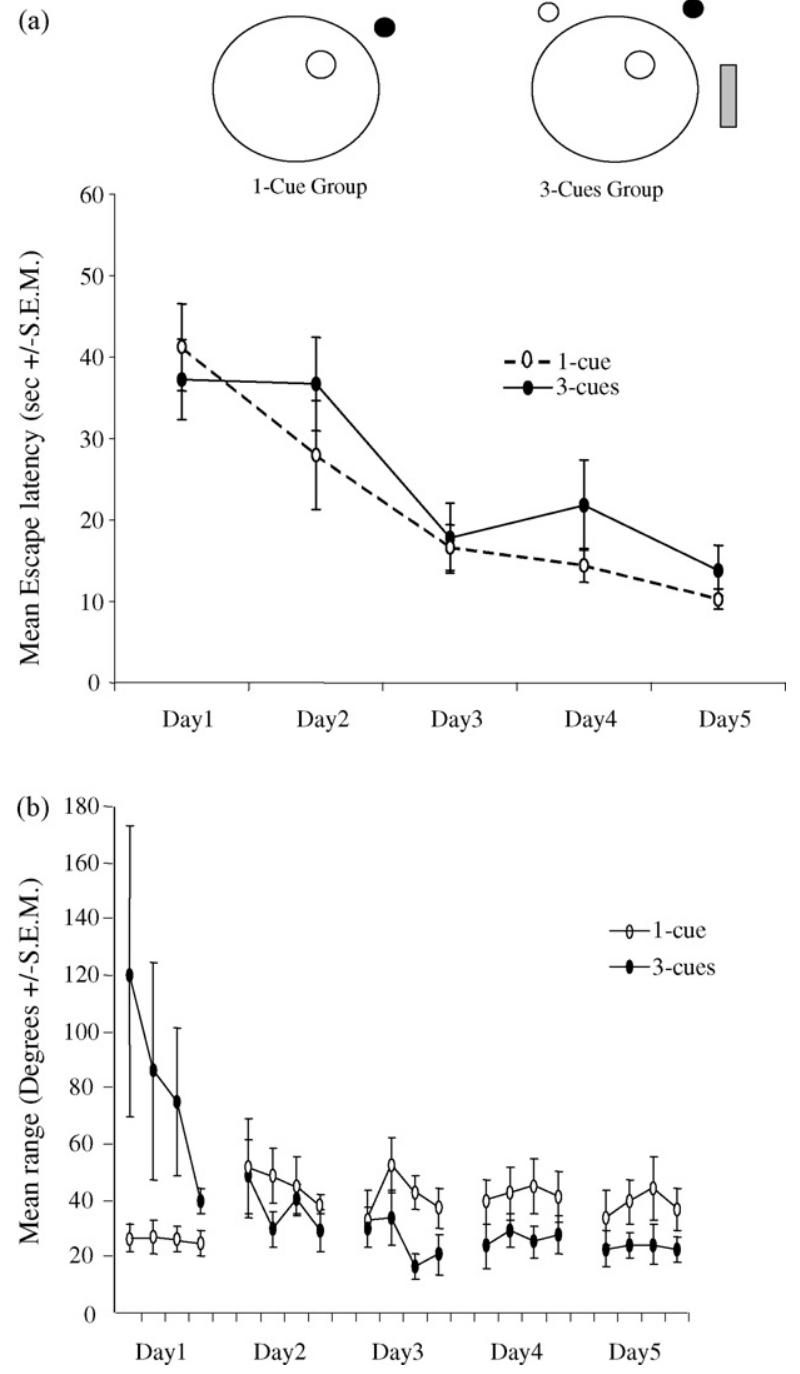

Fig. 1. (a) Mean escape latency ( \pm SEM) during acquisition. Inset: Schematic representation of the water maze set-up for both the 1-cue (left) and 3-cues groups (right). White and black circles outside the pool represent the 40 and $60 \mathrm{~W}$ light bulbs located in the NW and NE positions of the pool respectively with the grey rectangle representing the card located at the eastern position. (b) Mean range of head movement made $( \pm$ SEM) as the animal sits on the platform in between acquisition trials.

\subsection{Assessment of animals movement during trial}

Detailed examination of swimming behaviours was also conducted for each training trial across all experimental days, for all rats. For this, EthoVision provided $x, y$ coordinates $(0.2 \mathrm{~s}$ increments) for the animal's position throughout each trial. These tracks were then examined in depth. From this analysis and visual inspection a number of distinct swimming behaviours emerged. The first behaviour, scanning, was characterised by general forward movement by the animal while alternating body orientation from left to right. The minimum temporal episode permitted for this behaviour was $1 \mathrm{~s}$. A representative track illustrating this type of behaviour can been seen in Fig. 2a. Fig. 2a (i) illustrates the animal moving forward towards the rectangular sheet of paper (grey rectangle) in this scanning motion, keeping the cue generally centralised in its visual field. A similar behaviour has been documented in the solitary wasp (Cerceris; Zeil et al., 1996). The second, direct approach behaviour, was characterised by movements in a straight, definite direction over a minimum period of $1 \mathrm{~s}$. As we previously found that animals tended to move in the direction of the cues (Harvey et al.,
2008), the number of direct approaches to a cue-defined as the cue being maintained in a constant position in the animal's central visual field and the time spent engaged in this behaviour-was recorded. Fig. 2a (ii) illustrates a direct approach towards the light (white circle) and Fig. 2a (iii) illustrates direct approach towards the second light (black circle). This behaviour is comparable to the actions of the wood ant (Formica rufa; Judd and Collett, 1998) when approaching a landmark. More specifically when detailing rodents, this style of swimming has close similarities to the direct finding and approach behaviours defined by Graziano et al. (2003). The third behaviour is typified by a turn. For this, the animal moves in one direction followed by an obvious change in orientation (usually $>50^{\circ}$ ) and movement in a second direction. Therefore, "turning" is the episode of observable angular change between two periods of movement and in different directions. The turn is considered a whole body turn and not just the animal's head. In Fig. 2a, a number of turns are illustrated (T1) depicts a turn away from the rectangular sheet of paper and towards the light (white circle), (T2) depicts a turn towards the second light (black circle) and (T3) shows a turn away from the light cue and towards the platform.

\section{Results}

All animals successfully acquired the task. A $5 \times 2$ mixed factorial ANOVA revealed a significant effect for day $(F(4,56)=20.86$ $p<0.001)$. No interaction effects were revealed between the groups across days $(F(4,56)=1.032, p>0.05)$. Overall no differences were found between the groups' escape latency scores during acquisition $(F(1,14)=0.556, p>0.05)$ suggesting parallel learning of the task (Fig. 1a)

\subsection{Platform behaviour}

To investigate potential differences in the groups' behaviour on the platform during acquisition of the task, the platform interval was analysed. Firstly, the mean range viewed was assessed for each day. Following a $5 \times 2$ ANOVA, no main effect for day was found $(F(4,56)=2.45, p>0.05)$. No overall significant effect for group was revealed, suggesting both groups viewed a similar range from the hidden platform $(F(1,14)=0.56, p>0.05)$, however a day $\times$ group interaction was revealed $(F(4,56)=7.199, p<0.001)$. Independent $t$-tests indicated a significant difference between the mean range viewed in both groups only on Day $1(t(14)=2.78, p<0.05)$, with the 1 -cue group viewing a mean range of $26.03 \pm 4.86^{\circ}$ and the 3 -cues group viewing $80.13 \pm 29.85^{\circ}$ on this day (Fig. 1b). No other differences were noted. The direction in which animals faced on the platform was then investigated by calculating the mean head direction. Would animals in the 1-cue group orientate towards the single cue available? Rayleigh tests of uniformity indicated that there was no significant preferred head direction on any day for either the 3 -cues group or the 1 -cue group (all $p>0.05$ ).

\subsection{Characterisation of cue-dependent searching behaviours used during trial locomotion}

The swimming behaviour of animals in both groups during each trial was assessed in an attempt to identify subtle differences that may exist in how the groups acquire the task and how they use the cues. The first behaviour examined was direct approach. The mean number of direct approach behaviours for each animal in both groups was evaluated across training. Using a $5 \times 2$ ANOVA, a main effect for day was shown $(F(4,56)=3.41, p<0.05)$, whereby the 1 -cue group demonstrated a significant increase in the occurrences of this behaviour across training $(F(4,35)=2.756, p<0.05)$ but the 3 -cues group showed no alteration $(F(4,35)=1.657, p>0.05)$. An interaction effect was not identified $(F(4,56)=0.583, p>0.05)$. Similarly, 
(a)

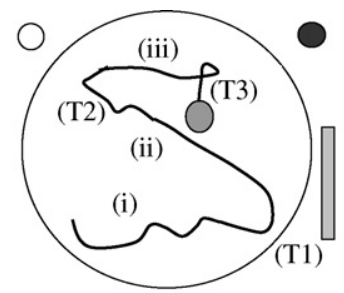

(b)

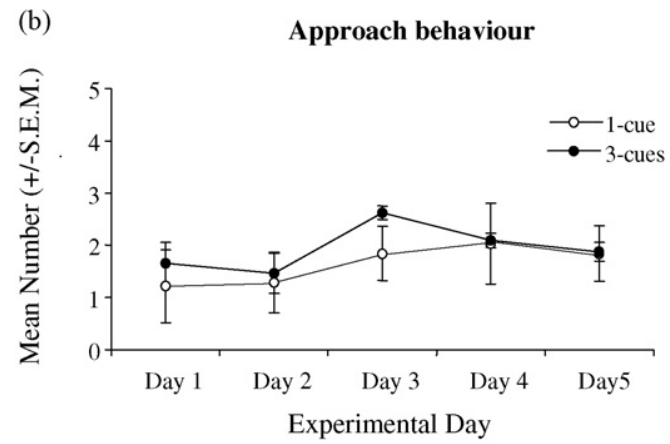

(d)

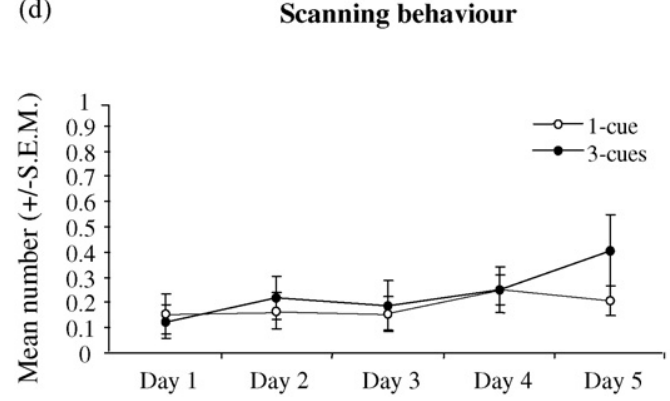

(c)
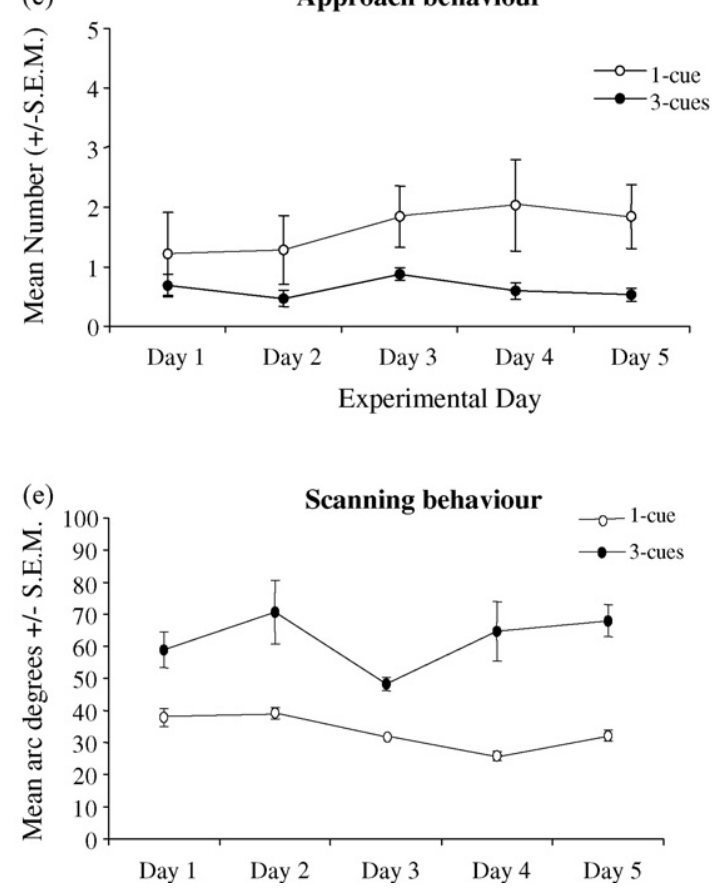

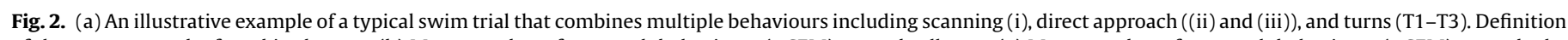

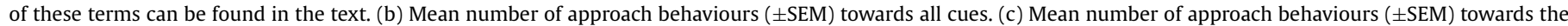

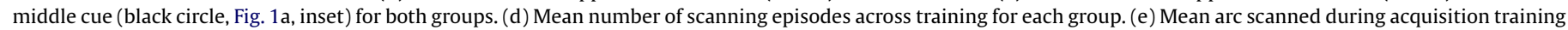
$( \pm$ SEM) for each group.

no main effect for group was found $(F(1,14)=2.54, p>0.05$; Fig. $2 b)$. This would suggest that the 1-cue group approached the single cue as frequently as the 3-cues group approached all the cues.

We then compared the approach of the 1-cue group to its cue to the approach of the 3-cues group to just the middle cue (this cue was located in the exact position as the single cue, black circle in Fig. 1a, inset). A main effect for day was found $(F(4,56)=2.769$, $p<0.05$ ). The 1 -cue group showed a significant increase in the mean number of approach episodes made during training as reported above. However, animals in the 3-cues group showed the same amount of this behaviour for the middle cue throughout training $(F(4,35)=1.291, p>0.05)$. A main group effect was also found $(F(1,14)=67.789, p<0.001$; Fig. $2 c)$ suggesting the 1 -cue group approached its cue more often than the 3-cues group approached its equivalent positional cue (middle cue). No interaction effect was revealed $(F(4,56)=2.034, p>0.05)$.

The second type of behaviour examined was scanning. The mean daily amount of scanning behaviour was assessed for each group with no main effect for day $(F(4,56)=0.933, p>0.05)$ or group $(F(1,14)=1.338, p>0.05)$ revealed (Fig. $2 d)$. Likewise no interaction effect was shown $(F(4,56)=0.817, p>0.05)$, suggesting that both groups used this behaviour to the same extent. However, when the mean arc scanned was investigated (that is, the arc covered as the animal moved leftwards and rightwards while continuing to locomote forward), differences were noted. While the $5 \times 2$ ANOVA exposed no main effect for day $(F(4,56)=0.213, p>0.05)$ or interaction effect $(F(4,56)=1.65, p>0.05)$, a main effect for group however was found (Fig. 2e, $F(1,14)=129.14, p<0.001$ ) suggesting that the 3 -cues group scanned a larger range on each day compared to the 1-cue group.

The third behaviour examined was when the animals changed their direction and turned away from the cues. As would be expected, we found a significant main effect for group $(F(1,14)=31.803, p<0.001)$; the 1 -cue group $(1.775 \pm 0.129)$ made significantly more turns away compared to the 3-cues group $(0.75 \pm 0.129 ; t(8)=4.575, p<0.001)$. No main effect for day $(F(4,56)=2.402, p>0.05)$, or interaction effect between day and group $(F(4,56)=2.478, p>0.05)$, was found. As there was a difference between the groups' overall amount of turning away, the spatial location of where the animals turned away from the cues was then examined. Overall it was observed that, in the 1-cue group, the location of where the animals turned away from the cues clustered in the NE of the pool, close to the provided distal cue (Fig. 3a, top panel). When daily Rayleigh tests of uniformity were carried out, significantly mean preferred locations on Days $1\left(98.39 \pm 20.50^{\circ}, Z=3.679 p<0.05\right), 3\left(137.88 \pm 16.59^{\circ}, Z=5.712\right.$, $p<0.01)$ and $5\left(85.21 \pm 18.93^{\circ}, Z=4.41, p<0.05\right)$ were identified (Fig. 3a, bottom panel, mean calculated from centre of platform). On the remaining days, however the mean directional location of this behaviour from the platform was randomly distributed around 

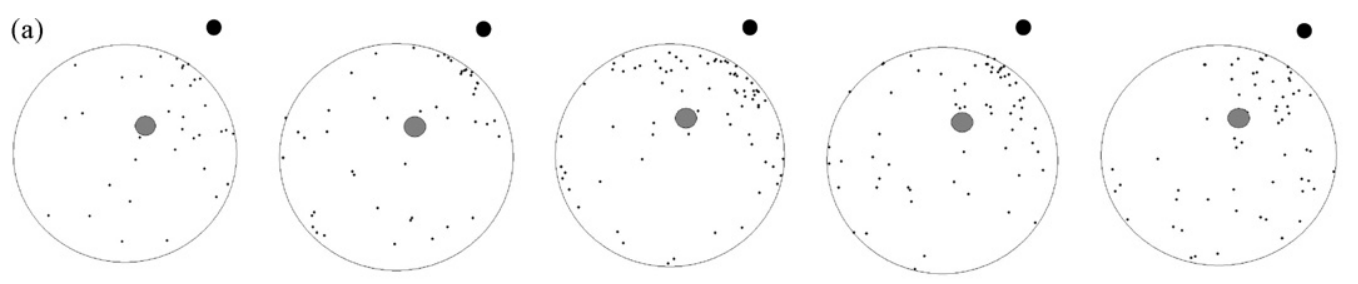

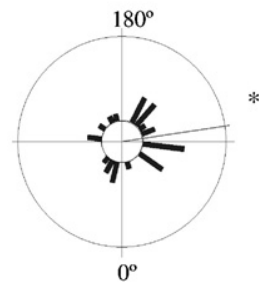

Day 1

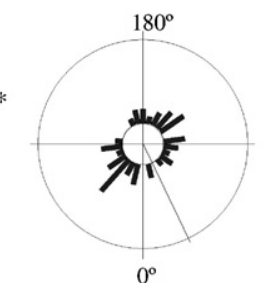

Day 2

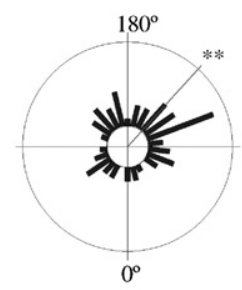

Day 3

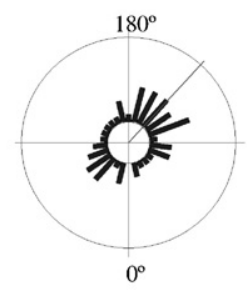

Day 4

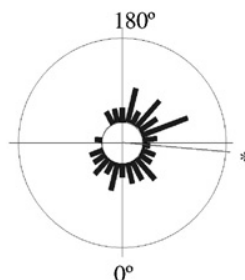

Day 5

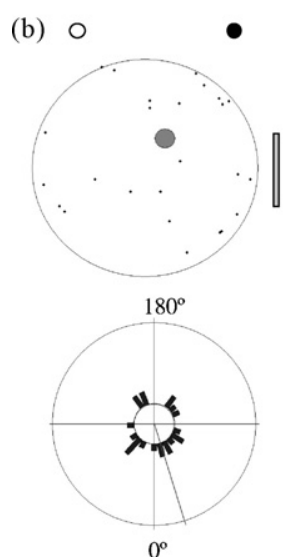

Day 1

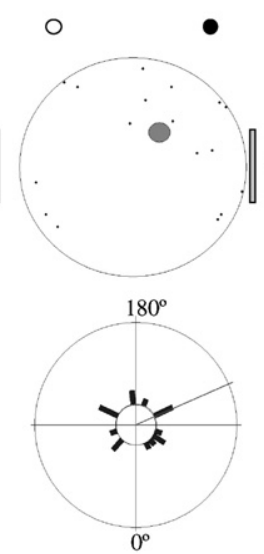

Day 2

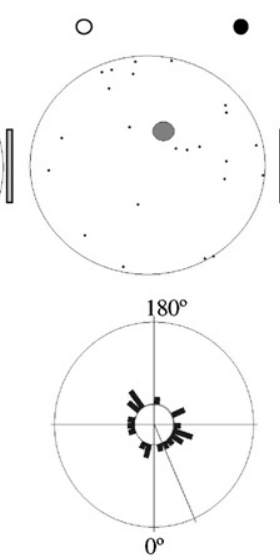

Day 3

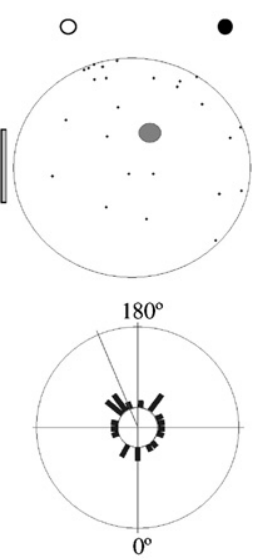

Day 4
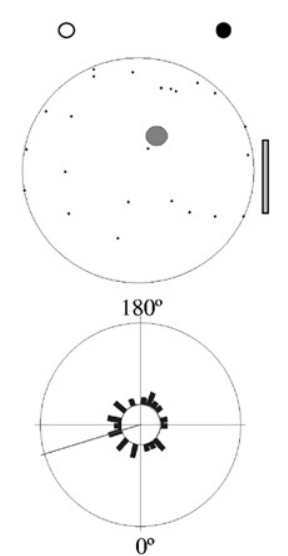

Day 5

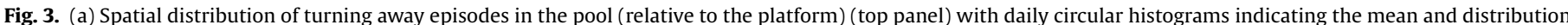

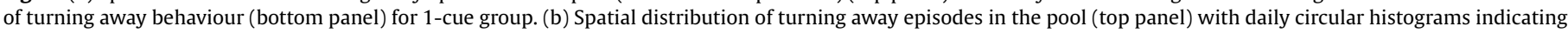
the mean and distribution of turning away behaviour (bottom panel) for 3-cues group. ${ }^{*} p<0.05$ and ${ }^{* *} p<0.01$.

the pool (all $p>0.05$ ). When the turn location of the 3-cues group was examined, more distributed patterns of overall turning away locations were identified (Fig. 3b, top panel), with daily Rayleigh uniformity tests confirming that there was no significantly preferred location for turning away on any day (all $p>0.05$; Fig. 3b, bottom panel). Therefore on the whole, turning away was initiated right throughout the pool in this group, rather than in preferred locations, as shown in the 1-cue group.

The final behaviour, turning towards the cues, was examined in both groups. The overall mean number of turns made daily was compared in each of the experimental groups. A main effect for day was found $(F(4,56)=9.46, p<0.001)$ with significantly more turns made by each group as training continued (3-cues group, $F(4,35)=2.51, p<0.05 ; 1$-cue group, $F(4,35)=7.195, p<0.001)$. A main effect for group was also shown $(F(1,14)=64.99, p<0.001)$ where the 3-cues group made more turns overall compared to the 1-cue group, as would be expected as this group had three rather than 1-cue to head towards. An interaction effect between day and group was also revealed $(F(4,56)=4.71, p<0.01)$. When we compared the mean number of turns made by the 3 -cues group to just the middle cue (i.e. the cue in the exact position as the single cue for the 1-cue group) to the turns of the 1-cue group towards its cue, a significant main effect for day was revealed $(F(4,56)=3.747$, $p<0.01$ ), with the 1 -cue group making significantly more turns with continued training $(F(4,35)=7.195, p<0.001)$; the 3 -cues group did not show any difference in the mean daily number of turns towards the middle cue $(F(4,35)=1.623, p>0.05)$. A main effect for group was also shown $(F(1,14)=7.273, p<0.05)$, indicating an overall difference between the two groups, with the 1-cue group making more turns overall towards their cue $(1.044 \pm 0.082)$ compared to the 3 -cues group $(0.731 \pm 0.082)$ therefore suggesting that, the 1 -cue group uses this cue more than the 3-cues group.

When the location of turns was examined in the 1-cue group (Fig. 4b, broken black line), a significant preferred location for turning was found on each training day (Day $1, Z=4.26, p<0.05$; Day 2, $Z=5.488, p<0.01$; Day 3, $Z=9.25, p<0.01$; Day $4, Z=5.92$, $p<0.01$; Day $5, Z=6.263, p<0.01$ ), all of which were to the $S$ of the platform (Fig. 4c, left panel). Further to this, the stability of the mean daily location for turns showed no significant differences following Watson's William F-tests, suggesting on each day turns were made in the same directional location from the platform (all $p>0.05$ ). We then assessed the preferred location of turns (towards each cue) for the 3-cues group. We found that turns towards the first light cue were all located in a significantly preferred position within the pool (SE of the platform) across each training day (open circle, Fig. 4b and c, right panel) (Day 1, $Z=4.179, p<0.01$; Day 2, $Z=5.365, p<0.01$; Day 3, $Z=4.8, p<0.01$; Day $4, Z=8.534$, $p<0.001$; Day $5, Z=7.739, p<0.001$ ). When the mean turn location was examined for the second light (black circle Fig. $4 \mathrm{~b}$ and $c$, right panel), significantly preferred locations were shown on Days $1(Z=3.279, p<0.05), 4(Z=3.061, p<0.05)$ and $5(Z=4.022, p<0.01)$. However turns for the rectangular card cue (grey circle, Fig. $4 \mathrm{~b}$ and 
(a)
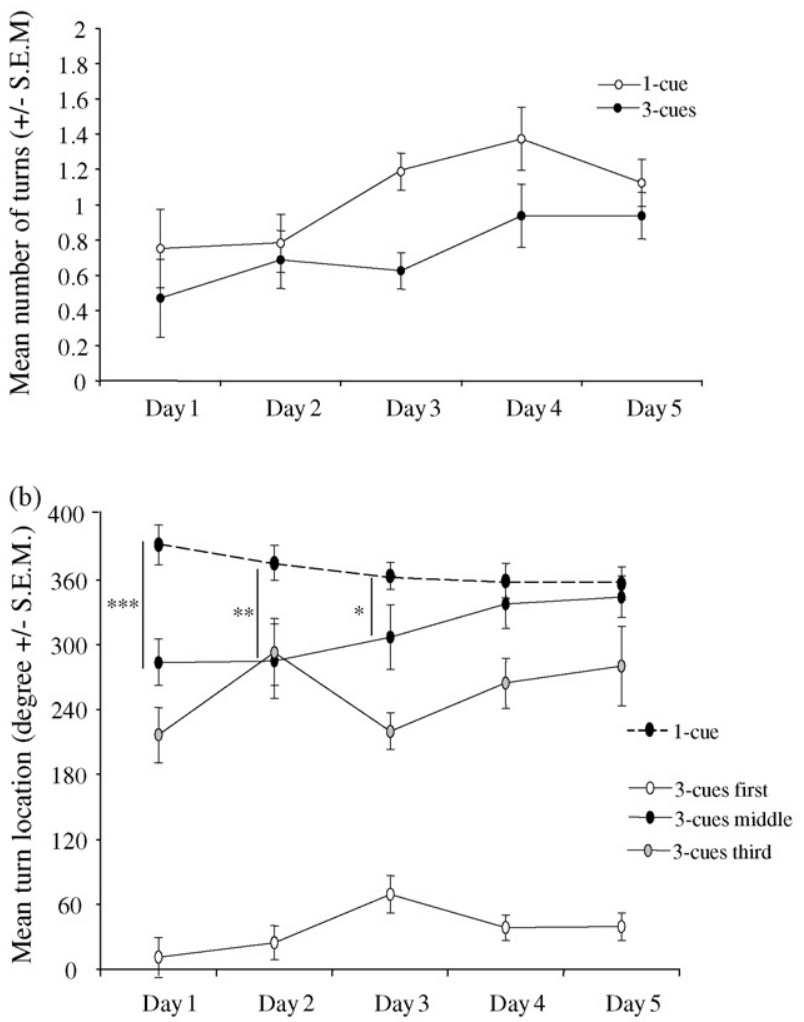

(c)
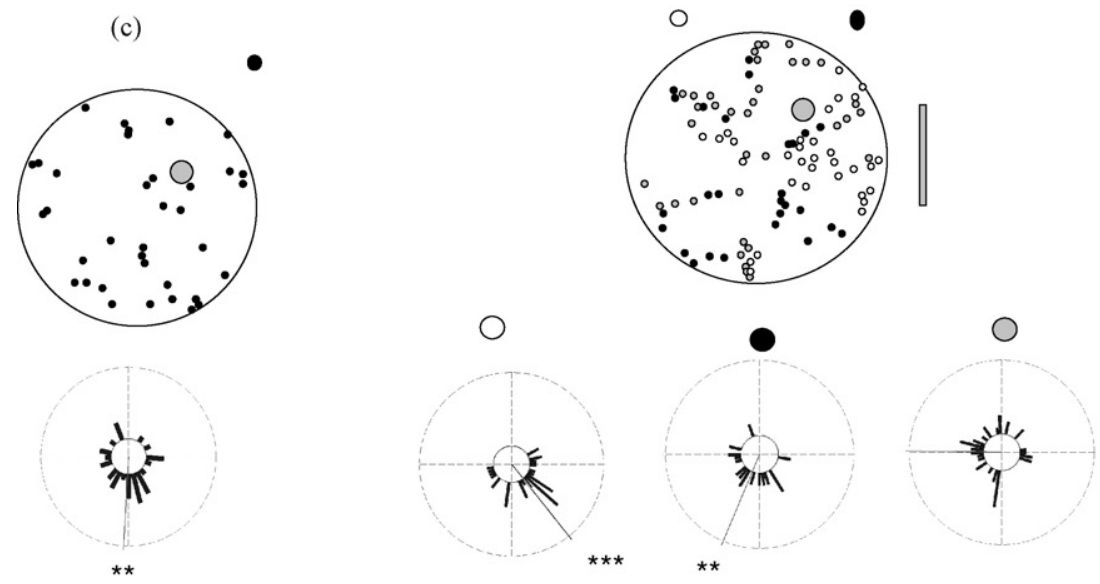

Fig.4. (a) Mean number of turns made $( \pm \mathrm{SEM})$ towards the cue during acquisition for 1 -cue group compared to the number of turns towards the middle cue for the 3 -cues group. (b) Alteration in turning behaviour during acquisition. Mean location of turns towards each cue $( \pm \mathrm{SEM})$ for both the one and three groups for each day. (c) Spatial distribution and circular histograms indicating the mean and distribution of turning episodes for Day 5 in the pool (relative to the platform) for 1-cue group (left panel) and 3-cues group (right panel; white, black and grey circles represent turns towards the first and second light cue, and the rectangular card respectively). ${ }^{*} p<0.05,{ }^{* *} p<0.01,{ }^{* * *} p<0.001$.

c, right panel) were only in a significantly preferred location on Day $3(Z=4.722, p<0.01)$. The stability of the preferred location of turns was also assessed for each respective cue across training. For the first light cue, Days 1 and 3 were significantly different $(F(1,37)=5.694, p<0.05)$ from each other. With respect to the second light cue, it was shown that the mean location of turns on Day 1 was significantly different to Days $4(F(1,38)=4.578, p<0.05)$ and $5(F(1,39)=5.312, p<0.05)$ only. Finally, when the mean location of turns for second light cue in the 3-cues group was compared with the 1-cue group significant difference in preferred turn location was found between the groups on Days $1-3(F(1,34)=15.013, p<0.001$; $F(1,31)=7.831, p<0.01 ; F(1,56)=5.036, p<0.05$ respectively, see Fig. $4 \mathrm{~b}$ ) but the preferred turn location on the remaining days was similar.

\section{Discussion}

The aim of this paper was to assess the effects of reduced cue availability on different swimming behaviours as animals acquire a spatial task. Reduced cue availability took the form of one distal visual cue placed outside the NE region of the MWM arena, whereas a second experimental group had three distal visual cues provided throughout training. Earlier studies have speculated that the number of available visual cues given can be influential on navigation (Fenton et al., 1994; Prados, 2000; Prados and Trobalon, 1998; Chamizo and Rodrigo, 2004; Collett et al., 1986; Martin et al., 2003; Roberts and Pearce, 1999).

The standard measure of acquisition (Escape Latency) did not reveal any differences between the groups overall, suggesting both 
groups acquired the task equally. This result is equivalent to that demonstrated by Martin et al. (2003), who showed animals trained to locate a goal with a single light cue were still able to acquire the goal's location, suggesting one cue can be used successfully. Indeed, in our experiment we cannot completely rule out the possibility that animals in the 1-cue group were using non-explicit cues to help them find the platform. A major non-explicit cue that animals could potentially use to gain distance information is the pool itself (Alvarado and Rudy, 1995), with recent evidence also suggesting that the shape of the pool and environment can contribute to an animal's ability to locate a goal (e.g. Hayward et al., 2004). Further to this, we have recently demonstrated that animals retested in a bright cue-free water maze environment (following training in a standard cue-filled environment) tend to swim both at the side of the pool in a thigmotaxic fashion and also around an inner corridor of the pool at a distance equivalent to the location of the platform relative to the pool's side. Animals in this group swam significantly more in this region compared to animals in a dark cue-less environment, which tended to remain at the side of the pool (Kealy et al., 2008; Experiment 3). These results suggest that animals can indeed use the pool wall to estimate distances. In the experiment presented here, animals in the 1-cue group had the benefit of the pool wall in addition to a distal cue, perhaps explaining the ability of animals in this group to readily acquire the task. Further, as the platform was always located at a fixed position from the pool wall, it could be argued that quite exact distance information can be gleaned from the wall. Potentially animals in the 1-cue group could therefore have found the platform very easily by using the simple strategy of swimming around the pool at the correct distance from the wall (similar to Kealy et al., 2008, described above). However, the use of our in-depth swimming analysis demonstrates that this is not the case and that animals in the 1-cue group use the light cue and the side of the pool in combination (see below).

The pattern of platform behaviour in the 3-cues group was similar to what we had previously reported (Harvey et al., 2008). Animals on the first trial, particularly on the first day of training tended to look around their environment, but by trial 4 the range observed decreased. The observed range stabilised across days. With the 1-cue group the range remained narrow and stable across the 5 days of training. Although the range of both groups remained consistent across time neither group looked in a preferred direction on any day. Although a single animal sitting on the platform may indeed have a preferred direction (particularly on the final trial of any given day), this animal may change its preferred direction across trials and days. We had predicted that animals trained with a single cue would tend to focus their attention on this cue. This prediction was however not upheld, perhaps suggesting that animals scan their environments rapidly and obtain basic spatial information. Indeed, we have previously demonstrated that animals having no cues available when sitting on the platform can acquire the task as readily as animals with a full set of cues available when on the platform (Harvey et al., 2008).

Our study confirms previous findings demonstrating the importance of the distal cues when navigating the water maze task (McGauran et al., 2004) and particularly demonstrates the reliance of animals on the cues. We show that modifying the number of cues modifies the searching behaviour of the animals. When animals are exposed to a single cue they become reliant on it for successful navigation. For example, animals in the 1-cue group approached this cue as much as the 3-cues group approached all cues. Similarly, animals in the 1-cue group tended to move in a scanning motion towards their cue as much as the 3-cues group scanned towards all cues, with scanning range in this group narrowing in line with the reduction in the availability of the cues. As the 1-cue group had only one cue by definition, this was the only reliable landmark providing assistance in swimming; therefore, if they were to sway too far from it when scanning, they could render themselves lost. The reliance on the single cue in the 1-cue group can be also observed by analysing the locations of turns made towards the cue. The animals in this group tended to turn south of the platform similar to the animals in the 3-cues group, however the mean location of turns for the 1-cue group remained very consistent and stable across the learning period. Whereas the mean turn location in the 3-cues group gradually shifted southwards with learning, perhaps suggesting that animals in this group are more flexible, being able to readjust their behaviour by using the other cues available if and whenever it was required for them to do so.

It has been demonstrated that trained animals using the water maze are more impaired when the cue is positioned on the wall on the opposite side of the pool than when it was positioned on the wall near the hidden platform (Chamizo and Rodrigo, 2004), but the animals' performance was best when the cue was suspended directly above the platform itself acting as a beacon (Gallistel, 1990). We would suggest that animals in the 1-cue group may also use their cue in a beacon-like fashion. We observed that animals in this group would turn towards the cue in the southern position of the pool (Fig. 3c) and then approach the cue, upon failing to reach the platform, animals would continue towards the edge of the pool adjacent to the cue and then turn back and reach the platform. This behaviour could well demonstrate an attempt to gain both direction and distance information using the cue and the pool's edge (see also Alvarado and Rudy, 1995). This suggestion is brought out by the greater number of incidences of turning away behaviour observed in this group that were significantly located in the NE position relative to the platform (Fig. 3a).

Finally, it has been suggested when two or more cues consistently occur together in predicting a given outcome, such as the location of a target position, less is learned overall about each of the individual cues than if it were one overall individual cue. In other words learning is shared between the cues (Shettleworth, 2005). Nevertheless, it has equally been suggested that in certain situations the use of only one cue can be favoured over a multiple cue configuration. This may be due to the individual cues' overbearing characteristics or dominant form, such as being louder, brighter, larger or indeed generally more salient than the alternatives available, consequently overshadowing the employment of multiple cues in the array (Shettleworth, 2005; Vallortigara et al., 1990). While we did not explicitly set out to examine competition between landmarks, we would agree with the sentiment that with multiple cues less is learned about each individual cue compared to a single available cue. We found that when the closest cue to the platform was assessed (middle cue) in the 3-cues group, animals used this cue significantly less across a range of behaviours (i.e. approach and stability, turning, scanning, turning away) when compared to the 1-cue group. In sum, the methodology employed in this paper may be usefully employed in the examination of general spatial learning strategies and may be further used to examine the influence of cue(s) on such strategies.

\section{References}

Alvarado, M.C., Rudy, J.W., 1995. Comparison of "configural" discrimination problems: implications for understanding the role of the hippocampus formation in learning and memory. Psychobiology 23, 178-184.

Benhamou, S., Poucet, B., 1998. Landmark use by navigating rats (Rattus norvegicus) contrasting geometric and featural information. J. Comp. Psychol. 112, 317-322.

Cartwright, B.A., Collett, T.S., 1983. Landmark learning in bees: experiments and models. J. Comp. Physiol. 151, 521-543.

Chamizo, V.D., Rodrigo, T., Peris, J.M., Grau, M., 2006. The influence of landmark salience in a navigation task: an additive effect between its components. J. Exp. Psychol.: Anim. Behav. Proc. 32, 339-344.

Chamizo, V.D., Rodrigo, T., 2004. Effect of absolute spatial proximity between a landmark and a goal. Learn. Motiv. 35, 102-114. 
Collett, T.S., Cartwright, B.A., Smith, B.A., 1986. Landmark learning and visuo-spatial memories in gerbils. J. Comp. Physiol. A 158, 835-851.

Fenton, A.A., Arolfo, M.P., Nerad, L., Bures, J., 1994. Place navigation in the Morris water maze under minimum and redundant extra-maze cue conditions. Behav. Neural Biol. 62, 178-189.

Gallistel, C.R., 1990. The Organization of Learning. MIT Press, Cambridge, MA.

Gallistel, C.R., Cheng, K., 1985. A modular sense of place? Behav. Brain Sci. 8, 11-12.

Graziano, A., Petrosini, L., Bartoletti, A., 2003. Automatic recognition of explorative strategies in the Morris water maze. J. Neurosci. Methods 130 (1), 33-44.

Harvey, D.R., McGauran, A.T., Murphy, J., Burns, L., McMonagle, E., Commins, S., 2008 Emergence of an egocentric cue guiding and allocentric inferring strategy that mirrors hippocampal brain-derived neurotrophic factor (BDNF) expression in the Morris water maze. Neurobiol. Learn. Mem. 89 (4), 462-479.

Hayward, A., Good, M.A., Pearce, J.M., 2004. Failure of a landmark to restrict spatial learning based on the shape of the environment. Q. J. Exp. Psychol. B 57 (4), 289-314.

Judd, S.P.D., Collett, T.S., 1998. Multiple stored views and landmark guidance in ants. Nature 392, 710-714.

Kealy, J., Diviney, M., Kehoe, E., McGonagle, V., O'Shea, A., Harvey, D., Commins, S. 2008. The effects of overtraining in the Morris water maze on allocentric and egocentric learning strategies in rats. Behav. Brain Res. 192, 259-263.

Martin, G.M., Walker, K.M., Skinner, D.M., 2003. A single unstable visual cue impairs spatial learning in a watermaze. Learn. Motiv. 34, 87-103.
Maurer, R., Derivaz, V., 2000. Rats in a transparent Morris water maze use elemental and configural geometry of landmarks as well as distance to the pool wall. Spatial Cog. Comp. 2, 135-156.

Morris, R., 1984. Developments of a water-maze procedure for studying spatial learning in the rat. J. Neurosci. Methods 11, 47-60.

Morris, R.G.M., 1981. Spatial localisation does not require the presence of local cues. Learn. Motiv. 12, 239-260.

McGauran, A.T., Harvey, D., Cunningham, L., Craig, S., Commins, S., 2004. Retention of cue-based associations in the water maze is time-dependent and sensitive to disruption by rotating the starting position. Behav. Brain Res. 151, 255-266.

Prados, J., 2000. Effects of varying the amount of preexposure to spatial cues on a subsequent navigation task. Q. J. Exp. Psychol. 53, 139-148.

Prados, J., Trobalon, J.B., 1998. Locating an invisible goal in a water maze requires at least two landmarks. Psychobiology 26 (1), 42-48.

Roberts, A.D.L., Pearce, J.M., 1999. Blocking in the Morris swimming pool. J. Exp. Psychol.: Anim. Behav. Proc. 25, 225-235.

Rodrigo, T., Chamizo, V.D., McLaren, I.P.L., Mackintosh, N.J., 1997. Blocking in the spatial domain. J. Exp. Psychol.: Anim. Behav. Proc. 23, 110-118.

Shettleworth, S.J., 2005. Taking the best for learning. Behav. Proc. 69, 147-149.

Spetch, M.L., 1995. Overshadowing in landmark learning: touch-screen studies with pigeons and humans. J. Exp. Psychol.: Anim. Behav. Proc. 21, 166-181.

Vallortigara, G., Zanforlin, M., Pasti, G., 1990. Geometric modules in animals' spatial representation: a test with chicks (Gallus gallus domesticus). J. Comp. Psychol. $104,248-254$. 